\title{
Systems analysis of apoptosis protein expression allows the case-specific prediction of cell death responsiveness of melanoma cells
}

\author{
E Passante ${ }^{1,2,4}$, ML Würstle ${ }^{1,2,4}$, CT Hellwig ${ }^{1,2}$, M Leverkus $^{3}$ and M Rehm ${ }^{\star, 1,2}$
}

Many cancer entities and their associated cell line models are highly heterogeneous in their responsiveness to apoptosis inducers and, despite a detailed understanding of the underlying signaling networks, cell death susceptibility currently cannot be predicted reliably from protein expression profiles. Here, we demonstrate that an integration of quantitative apoptosis protein expression data with pathway knowledge can predict the cell death responsiveness of melanoma cell lines. By a total of 612 measurements, we determined the absolute expression $(\mathrm{nM})$ of 17 core apoptosis regulators in a panel of 11 melanoma cell lines, and enriched these data with systems-level information on apoptosis pathway topology. By applying multivariate statistical analysis and multi-dimensional pattern recognition algorithms, the responsiveness of individual cell lines to tumor necrosis factor-related apoptosis-inducing ligand (TRAIL) or dacarbazine (DTIC) could be predicted with very high accuracy (91 and $82 \%$ correct predictions), and the most effective treatment option for individual cell lines could be pre-determined in silico. In contrast, cell death responsiveness was poorly predicted when not taking knowledge on protein-protein interactions into account (55 and $36 \%$ correct predictions). We also generated mathematical predictions on whether anti-apoptotic Bcl-2 family members or $x$-linked inhibitor of apoptosis protein (XIAP) can be targeted to enhance TRAIL responsiveness in individual cell lines. Subsequent experiments, making use of pharmacological Bcl-2/Bcl-xL inhibition or siRNA-based XIAP depletion, confirmed the accuracy of these predictions. We therefore demonstrate that cell death responsiveness to TRAIL or DTIC can be predicted reliably in a large number of melanoma cell lines when investigating expression patterns of apoptosis regulators in the context of their network-level interplay. The capacity to predict responsiveness at the cellular level may contribute to personalizing anti-cancer treatments in the future.

Cell Death and Differentiation (2013) 20, 1521-1531; doi:10.1038/cdd.2013.106; published online 9 August 2013

Many cancer entities are biologically highly heterogeneous. As a consequence of biological heterogeneity, only subsets of patient populations benefit from anti-cancer chemotherapies. On an average, only $15 \%$ of metastatic melanoma patients respond to the standard single-agent chemotherapy with the proapoptotic alcylating agent dacarbazine (DTIC). ${ }^{1}$ Likewise, alternative chemotherapeutics or DTIC combination treatments so far did not result in more effective therapies. ${ }^{1}$ Interindividual heterogeneity also contributes to the unsustainably high attrition rates of phase II/II clinical trials, as cohort recruitment is often not informed by tools that allow to predict whether patients are likely to respond to treatment. ${ }^{2}$ Biological heterogeneity has been captured in numerous biomarker discovery studies to identify whether molecular signatures can predict drug responsiveness. However, these traditional statistical approaches so far are rather unsuccessful, with most biomarker candidates failing at the clinical validation stage. ${ }^{3}$ In melanoma, the presence of V600E-mutated B-Raf kinase is such a marker, and can be found in approximately $50 \%$ of patients with advanced disease. Whenever V600Emutated B-Raf is found, the inhibitor Vemurafenib has proved to be a remarkable success as monotherapy. ${ }^{4}$ The remaining patient population, however, still receives DTIC-based chemotherapy or immune therapy with ipilimumab, a CTLA4 antagonizing antibody. Most recently combination therapies of programmed death-1 receptor-directed antibodies combined with ipilimumab showed a dramatic success, indicating that immune-mediated cell death is a successful way to control melanoma metastasis. ${ }^{5}$ Even though deregulations in apoptosis signaling are known to limit melanoma responsiveness to treatment, ${ }^{6}$ so far no apoptosis-related protein expression patterns could be firmly associated with responsiveness to DTIC or other apoptosis-inducing agents in patients or cell line model systems. A broader

\footnotetext{
${ }^{1}$ Department of Physiology and Medical Physics, Royal College of Surgeons in Ireland, Dublin 0002, Ireland; ${ }^{2}$ Centre for Systems Medicine, Royal College of Surgeons in Ireland, Dublin 0002, Ireland and ${ }^{3}$ Section of Molecular Dermatology, Department of Dermatology, Venereology, and Allergology, Medical Faculty Mannheim, University of Heidelberg, Heidelberg, Germany

*Corresponding author: M Rehm, Department of Physiology and Medical Physics, Royal College of Surgeons in Ireland, RCSI York House, York Street, Dublin 2, Ireland. Tel: + 3531402 8563; Fax: + 3531402 2447; E-mail: mrehm @ rcsi.ie

${ }^{4}$ These authors contributed equally to this work.

Keywords: systems biology; apoptosis; melanoma; cancer; TRAlL

Abbreviations: cyt-c, cytochrome-c; DISC, death-inducing signaling complex; DTIC, dacarbazine; LDA, linear discriminant analysis; LOOCV, leave-one-out crossvalidation; PCA, principal component analysis; PC, principal component; TRAIL, tumor necrosis factor-related apoptosis-inducing ligand; XIAP, x-linked inhibitor of apoptosis protein

Received 31.1.13; revised 16.6.13; accepted 01.7.13; Edited by B Zhivotovsky; published online 09.8.13
} 
multi-parametric system-level approach toward apoptosis signaling in cell line models is therefore warranted and may contribute to informing future translational research strategies. Apoptosis signals are transduced by two main pathways (extrinsic and intrinsic), which are tightly regulated by a complex interplay of multiple proteins. ${ }^{7,8}$ Therefore, a large number of possibilities exist by which apoptosis signaling may be impaired. Integrating information on protein-protein interplay and pathway topology into data sets on protein expression may therefore allow multivariate statistical models to accurately and case-specifically predict cell death responsiveness. The perspectives offered by systems biological and systems medical approaches, ${ }^{9}$ and the first successful proofof-principle systems medical studies in the field of cancer and apoptosis ${ }^{10,11}$ support this notion.

Here, we therefore developed a system-level approach of knowledge- and data-driven multivariate statistical modeling and applied this to a panel of malignant human melanoma cell lines in order to identify whether baseline protein expression patterns carry information on the susceptibility to apoptosisinducing drugs.

\section{Results}

Defining functional groups of apoptosis proteins for a knowledge- and data-driven modeling approach. As decision points in apoptosis signal transduction are under the control of multi-protein interactions, we integrated basic pathway knowledge on protein-protein interplay into baseline expression data. This was achieved by defining functional groups, each of which combined a limited number of proteins by arithmetic operations. These operations reflected the protein relationship within the signaling network that is initiated by tumor necrosis factor-related apoptosis-inducing ligand (TRAIL; Figure 1a). In brief, TRAIL binds to death receptors DR4 and DR5. DR4/5 then recruit the adapter protein FADD and form the death-inducing signaling complex (DISC). Caspase-8 is activated on the DISC, but activation can be antagonized by the catalytically inactive homolog cFLIP. Caspase-8 proteolytically activates the BH3-only protein Bid as well as effector caspase-3. Truncated Bid promotes the permeabilization of the outer mitochondrial membrane by activating Bax and Bak, and by inhibiting antiapoptotic Bcl-2 family members Bcl-2, Bcl-xL and $\mathrm{Mcl}-1$. Cytochrome-c (cyt-c) and the $x$-linked inhibitor of apoptosis protein (XIAP) antagonist Smac are released into the cytosol. Cyt-c activates Apaf-1, and Apaf-1 oligomerizes to form the caspase-9-activating apoptosome. Caspase-9 proteolytically activates caspase-3, with the inhibitor XIAP suppressing caspase activity. Caspase-3 cleaves a large number of target proteins and is the central driver of rapid apoptosis execution. ${ }^{7,8}$ The intrinsic apoptosis pathway is induced by various forms of intracellular stresses and feeds in at the level of BH3-only proteins, which are transcriptionally induced, posttranslationally activated or stabilized, and contribute as Bax/Bak activators and/or as Bcl-2/Bcl-xL/ Mcl-1 antagonists.

To code this pathway by functional groups, the rationale was to keep these groupings arithmetically simple (sums, products or ratios of a small number of proteins), biologically justifiable and to incorporate the amount of each protein only once (Figure 1b). For example, we defined a functional group that represents the key anti-apoptotic Bcl-2 family members by pooling the absolute amounts (nM) of Bcl-2, Bcl-xL and $\mathrm{Mcl}-1$. Likewise, we defined the mitochondrial Bax/Bak pore formation capacity by pooling the amounts of Bax and Bak. By multiplying Apaf- 1 and caspase- 9 , we defined a functional group that represents the caspase-9-activating apoptosome complex. Here, the multiplication ensures that the value for this group approaches zero when either Apaf-1 or caspase- 9 expression is very low or absent. With similar reasoning, we multiplied the pooled DR expression with the amount of FADD to represent the DISC. Functional groups of apoptotic caspases and their antagonists were defined as ratios. Following these groupings, the three remaining proteins (Bid, cyt-c, Smac) were kept as individual variables. Although cyt-c is known to be an abundant protein, ${ }^{12}$ only a fraction of the cyt-c pool would be expected to contribute to apoptosis signaling. For example, significant amounts of cyt-c may be immobilized by cardiolipin interactions ${ }^{13,14}$ or may otherwise be inaccessible for apoptosome formation. ${ }^{15-17}$ Other BH3only proteins or Omi/HtrA2, which were not quantified in this study but functionally overlap with Bid or Smac, could form functional groups with the latter in future model extensions.

To parameterize the functional groups, we measured the expression of the 17 key proteins involved in TRAIL-induced apoptosis in 11 melanoma cell lines. With the exception of DR4/5, whose cell surface expressions were determined by indirect immunofluorescence (Supplementary Figure 1A), all amounts were measured by quantitative immunoblotting at 12-bit dynamic range (absolute protein concentrations are provided in Supplementary Table 1). Representative 8-bit converted signals are shown in Supplementary Figure 1B. An overview based on 612 quantifications is presented as Figure 1c and demonstrates the high heterogeneity in protein expression across the cell line panel.

Multi-dimensional pattern recognition identifies a relation between apoptosis regulators and cell death responsiveness to TRAIL and DTIC. As part of a datadriven modeling approach, ${ }^{18}$ we next applied a principal component analysis (PCA) ${ }^{19,20}$ to the data set of the functional groups and combined this with the integration of drug response data into the PCA output and a spatial segmentation of cell line clusters with similar drug responsiveness (see step-by-step workflow in Supplementary Figure 2). A PCA is a multivariate statistical procedure that reduces the dimensionality of complex multi-dimensional data sets by mathematical transformation into principal components (PCs). This transformation can be understood as a rotation of the data around their mean so that previously independent variables (the nine dimensions of the functional groups; Figure 1b) now jointly define new axes. These new axes are the PCs and describe the PC space. Although each $\mathrm{PC}$ consists of contributions by all variables of the original data set, the contributions of these variables differ for each $\mathrm{PC}$ according to specific weighting coefficients, as shown below. The PCA is geared toward representing as much of the data variance as possible in the first few PCs, thereby making the information content of the remaining PCs 
a

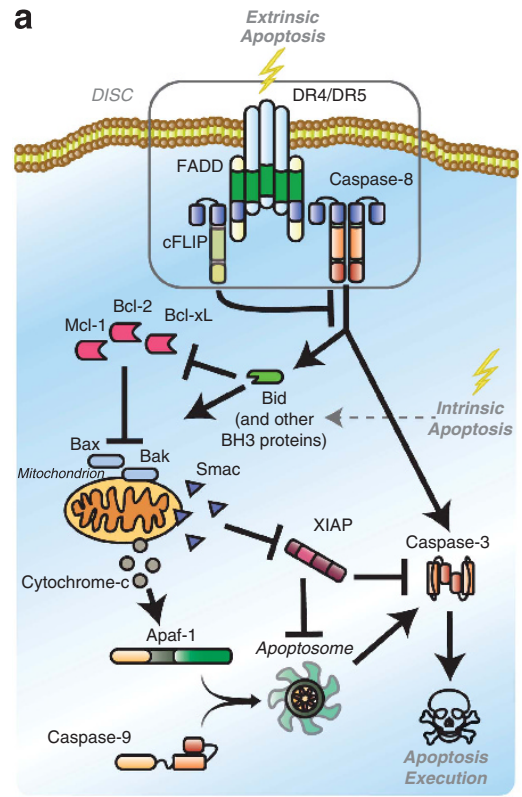

b

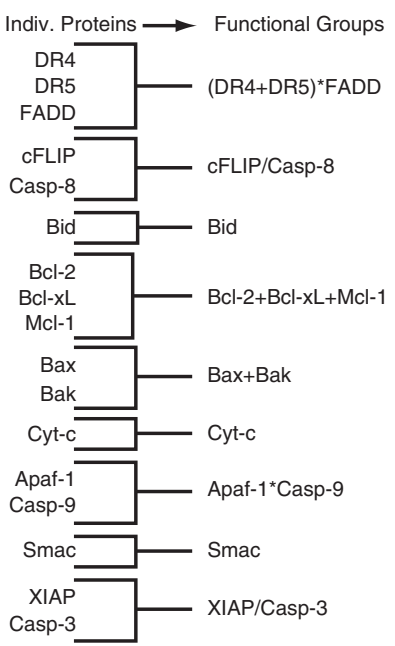

C

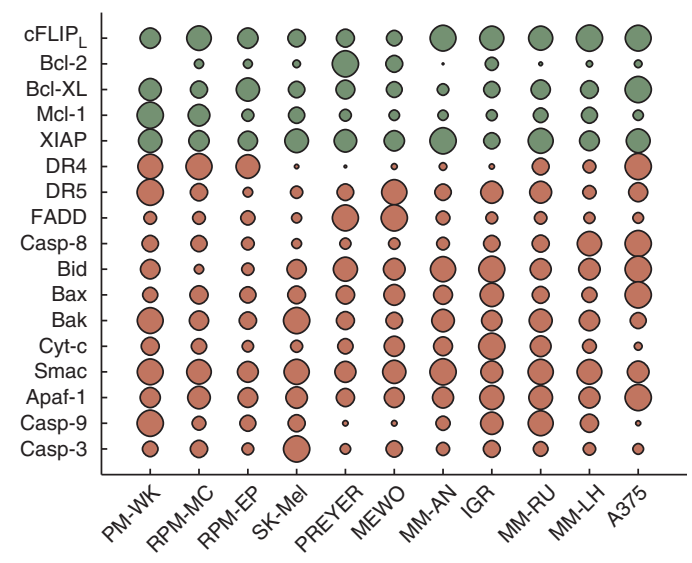

Figure 1 Coding and parameterizing functional groups of apoptosis signal proteins for knowledge- and data-driven systems modeling. (a) Pathway diagram of extrinsic apoptosis signal transduction through the TRAIL pathway. (b) Combination of apoptosis signaling proteins into functional groups by simple arithmetic operations. (c) Relative levels of protein expression within the melanoma cell line panel. Circles summarize 612 quantifications and circle sizes are proportional to the protein quantities determined from $n=3$ independent detections each. Green and red indicate anti- and pro-apoptotic proteins, respectively. Absolute protein amounts are provided in Supplementary Table 1

negligible for further analyses. We visualized this by showing the PCA results in a modified scree plot (Figure 2a). The first four PCs were required to reflect $85 \%$ of the data variance found in the functional groups, and these four PCs fulfilled the Kaiser criterion ${ }^{21,22}$ (Figure 2a). The Kaiser criterion is a decision guideline, which states that only PCs with an eigenvalue of at least one are needed for further analysis as these PCs contain more information on data variance than a single variable in the original data set. The first four PCs were therefore retained for all subsequent analyses. The associated weighting coefficients for the functional groups are displayed in Figure $2 \mathrm{~b}$ and are also provided in Supplementary Table 2. The coefficients indicated that the first PC is strongly influenced by multiple functional groups: the anti-apoptotic $\mathrm{Bcl}-2$ proteins are antagonized by $\mathrm{Bax}+$ Bak. Similarly, contributions by the XIAP/caspase-3 ratio are antagonized by caspase-9*Apaf- 1 . (DR4 +5$)^{*} \mathrm{FADD}$ and Bid strongly contribute to the second PC, as is cyt-c. The cFLIP/ casp-8 ratio prominently influences the fourth PC. We used the PCs to define a four-dimensional Euclidean space into which we positioned the 11 cell lines according to their functional groups values. To this end, these values were multiplied with the respective weighting coefficients in the PCs. Associated visualizations naturally had to be limited to the first three dimensions, which still reflect $71 \%$ of the original data variance (Figure 2c). Cell lines with similar compositions of the functional groups would have been expected to cluster together. However, no obvious clusters could be detected. Rather, cell lines were scattered throughout the PC space (Figure 2c), indicating a high variability in the values of the functional groups across the cell line panel.
We next investigated whether the cell line positions are related to their cell death responsiveness. To this end, we measured the overall cell death induced by TRAIL or DTIC, the standard melanoma chemotherapeutic in B-Raf-negative melanoma. DTIC is a pro-drug that is converted intracellularly by mitochondrial cytochrome P450 to the alkylating agent MTIC, and induces apoptosis through the intrinsic pathway. ${ }^{23}$ Cell death in response to TRAIL or DTIC was highly heterogeneous across the cell line panel, and individual cell lines typically were also differentially sensitive to TRAIL or DTIC (Figure 2d). Caspase inhibition in highly responsive cell lines demonstrated that cell death was executed primarily by apoptosis (Supplementary Figure 3). The cell lines in the PC space were then color coded according to their TRAIL or DTIC responsiveness (Figures $2 e$ and $f$ ). Cell lines were defined as resistant $(0-10 \%$ cell death; black) or responsive (graded into 'low' (10-30\% cell death; red), 'medium' (30-60\% cell death; yellow) or 'high' ( $>60 \%$ cell death; green)). Interestingly, cell lines with a similar TRAIL responsiveness appeared to occupy common regions in the 3D PC space (Figure 2e), and these response regions did not intersect when visually segmented (see also $360^{\circ}$ rotation in Supplementary Movie 1). Common response regions were also found for DTIC treatments (Figure 2f; see also $360^{\circ}$ rotation in Supplementary Movie 2 and an alternative viewing angle provided as Supplementary Figure 4). Corresponding to the cell line panel responding differently to TRAIL or DTIC, the spatial response regions differed between TRAIL and DTIC treatments (Figures $2 \mathrm{e}$ and f; Supplementary Movies 1,2). To determine whether the spatial regions could also be separated objectively, we applied a linear discriminant analysis (LDA) to the four-dimensional PC space. A canonical LDA is a pattern 


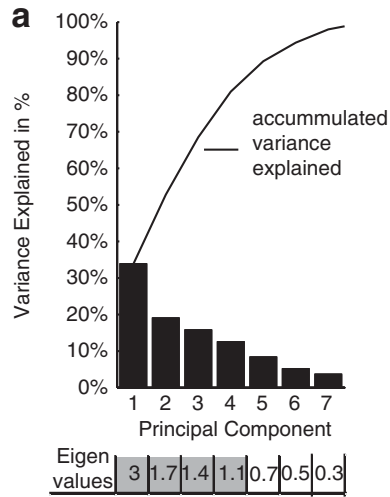

b

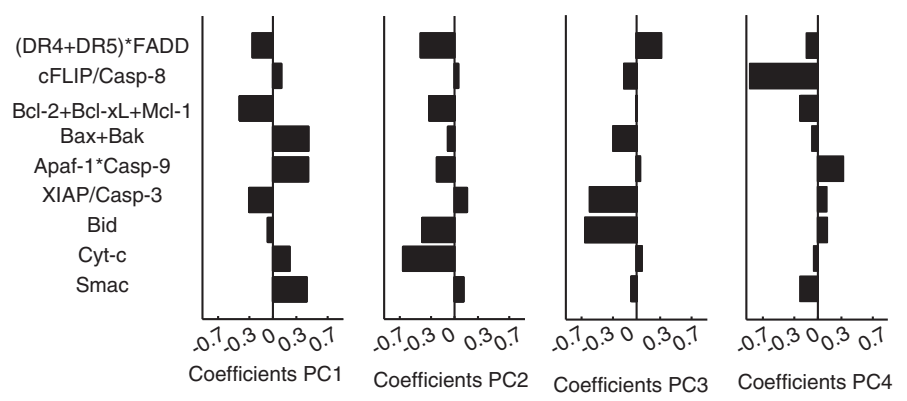

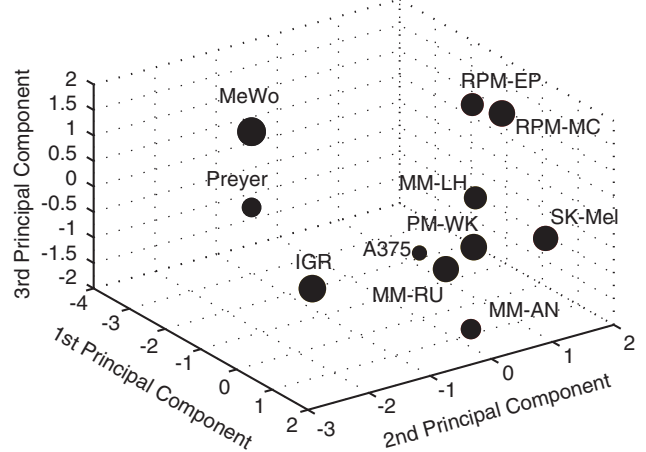

d

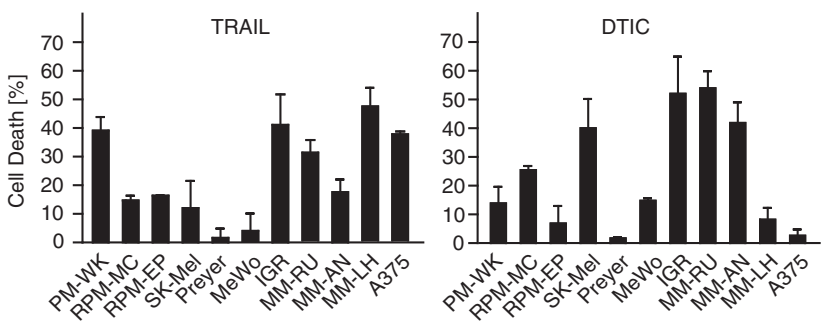

e

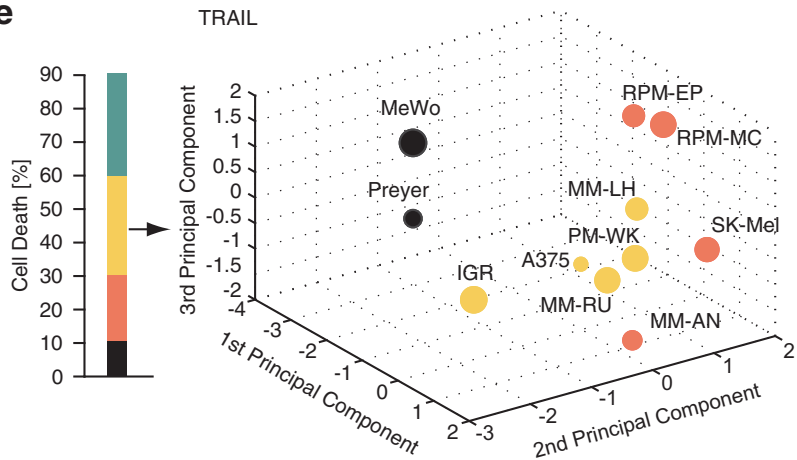

g

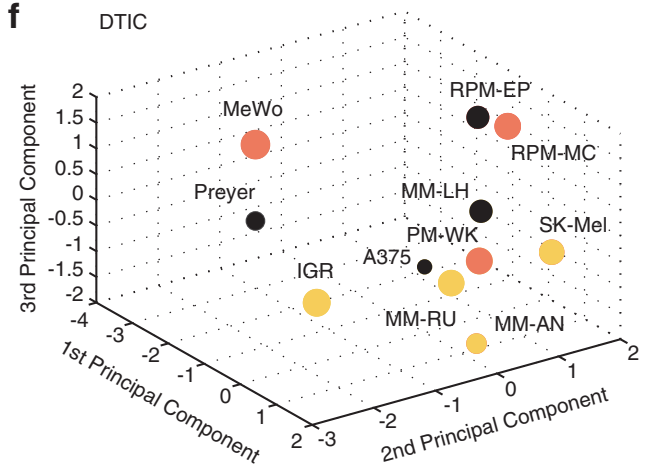

h

\begin{tabular}{c|c|c} 
Drug & $\begin{array}{c}\text { Correctly } \\
\text { classified \# }\end{array}$ & $\begin{array}{c}\text { Correctly } \\
\text { classified in \% }\end{array}$ \\
\hline TRAIL & 11 & $100 \%$ \\
DTIC & 10 & $91 \%$
\end{tabular}

Figure 2 Functional groups of apoptosis regulators highlight an association between protein expression profiles and cell death responsiveness to TRAIL and DTIC. (a) A principal component analysis (PCA) was applied to the functional groups of the melanoma cell line panel, and results are shown as a scree plot. Bar graphs show the contribution of each principal component (PC) toward explaining the data variance. Eigenvalues of PCs fulfilling the Kaiser criterion are shaded in gray. (b) Bar graphs show the coefficients for all functional groups in the first four PCs. Coefficient values are provided as Supplementary Table 2. (c) Graphical illustration of the distribution of melanoma cell lines along the first three PCs. Circle sizes decrease with distance from the observer to aid 3D visualization. (d) Cell death in response to TRAIL or DTIC. Cells were treated for $48 \mathrm{~h}$ and cell death was measured by propidium iodide uptake. Data are means + S.D. above untreated controls and were pooled from $n=3$ independent repeat experiments. (e and f) Cell lines in the 3D PC space were color coded according to cell death responsiveness to TRAIL or DTIC, respectively. Color codes reflect resistance (black), low responsiveness (red) or medium responsiveness (yellow). Cell lines with high responsiveness (green) were not detected. 3D rotations are available as Supplementary Movies 1 and 2. (g) Linear discriminant analysis (LDA) was applied to the four-dimensional PC space. The 2D schematic visualizes the principle of separating response regions by this pattern recognition approach. (h) The performance of the response group separation in the four-dimensional space by LDA is shown by listing the amounts of correctly classified cell lines 
recognition approach that defines linear functions with the aim to optimally separate multiple classes of objects in $n$ dimensional space. ${ }^{24}$ The LDA working principle is visualized for a simplified 2D scenario in Figure 2g. LDA accurately separated the TRAIL response groups. All cell lines were correctly classified (Figure 2h). Likewise, a very good separation was achieved for DTIC, with 10 out of 11 cell lines correctly classified (91\%; Figure $2 \mathrm{~h}$ ).

Taken together, we found that spatial regions exist, which represent distinct levels of drug responsiveness, that these spatial regions differ between TRAIL and DTIC treatments, and that these regions can be separated by a pattern recognition algorithm. Therefore, even though the cell lines are very heterogeneous in their protein expression profiles, these protein profiles, together with basic pathway knowledge, can be used to relate expression patterns to cell death susceptibility with high accuracy.

Case-specific predictions of TRAIL and DTIC responsiveness allow the in silico identification of optimal treatments. To determine whether cell death responses can be predicted case and drug specifically, we next applied 'leave-one-out' cross-validations (LOOCV). To this end, 11 new PCAs were conducted to cover all possible combinations of 10 from the pool of melanoma cell lines. Clusters of common responsiveness were then identified by LDA as described above. The missing cell lines (test cell lines) were positioned into the four-dimensional PC spaces, and their responsiveness was predicted based on the LDA-defined cluster associated with their PC space position. These predictions were validated against experimentally measured cell death responses. 2D projections of 3D PC spaces for TRAIL responsiveness demonstrate that test cell lines positioned in close proximity to cell lines with similar responsiveness (Figure 3a). The predictive power of this approach was very high for both TRAIL and DTIC treatments, with 10 and 9 cell lines positioning in the correct response regions ( $91 \%$ and $82 \%$ accuracy, respectively; Figure 3b). As a control, we performed the same procedure on the raw protein expression data rather than the functional groups data. The accuracy dropped significantly, with the responsiveness of only $55 \%$ and $36 \%$ of the cell lines correctly predicted for TRAIL or DTIC treatments, respectively. This highlights that accurate predictions can only be made when taking pathway knowledge into account.

We next investigated whether this predictive capacity is sufficiently high to case specifically determine the optimal treatment in silico. 'Optimal treatment' here is defined as the drug that evokes the higher amount of cell death, with either drug being an acceptable choice if both were predicted to induce comparable amounts of cell death. In contrast, wrong predictions suggest treatments that in experiments perform worse than the alternative drug. Cell line-specific suggestions were correct for 10 out of the 11 cell lines (Figure 4). Only for PM-WK cells, the better treatment option was not identified. These results indicate that our approach can predict cellular drug responsiveness and can select the optimal treatment option between TRAIL and DTIC for individual cell lines.
Systems modeling can identify targeted perturbations that sensitize poorly responding cell lines to TRAIL. The positioning of each cell line in the PC space is coded by the values calculated for their functional groups. We therefore hypothesized that this information could be exploited to generate case-specific predictions on how to sensitize poor TRAIL responders by targeted drugs or by siRNA-based protein depletion. As a representative targeted drug, we used ABT-737, a well-characterized synthetic antagonist of Bcl-2 and $\mathrm{BCl}-\mathrm{xL}^{25}$ that is currently also clinically tested as a sensitizer of melanoma to proliferation inhibitors such as MEK inhibitors. ${ }^{26}$ To generate predictions on which poor responders can be sensitized by ABT-737, we determined how their position in the PC space would change upon elimination of $\mathrm{Bcl}-2$ and $\mathrm{Bcl}-\mathrm{xL}$. The vector for the direction of this repositioning can be calculated from the PCA results by moving in opposite direction to the coefficients of the targeted functional group in all PC axes (Figure 5a). The distance by which individual cell lines are repositioned then depends on the combined amounts of its targets $(\mathrm{Bcl}-2+\mathrm{Bcl}-\mathrm{xL}$; Figure $5 b$ ). The resulting repositioning vectors were applied to four representative cell lines that poorly respond to TRAIL (RPM-EP, RPM-MC, MeWo, Preyer). For the TRAIL-resistant MeWo and Preyer cells, the vectors pointed in the direction of cell lines that are moderately TRAIL sensitive (yellow; Figure 5c), indicating that addition of ABT-737 may enhance TRAIL responsiveness. In contrast, for the poor TRAIL responders RPM-EP or RPM-MC the vectors did not result in a movement toward regions of higher TRAIL responsiveness (Figure $5 \mathrm{c}$ ). The predictions on high versus low sensitization by ABT-737 were validated experimentally. ABT-737 strongly sensitized MeWo and Preyer cells to TRAIL, whereas sensitization of RPM-EP and RPM-MC was far less pronounced (Figure 5d). Control experiments ensured that ABT-737 readily entered all cell lines and sensitized these for mitochondrial translocation of ectopically expressed YFP-Bax (Supplementary Figure 5). We next applied the same approach to a scenario of siRNA-mediated XIAP depletion. Our calculations on movement direction and movement distance (Figures $5 e$ and f) suggested that out of four poor TRAIL responders (Preyer, MeWo, RPM-EP, MM-AN) only MM-AN could be relocated closer to cell lines with higher TRAIL responsiveness (Figure $5 \mathrm{~g}$ ). Again, these predictions were confirmed by subsequent experiments. Even though XIAP was efficiently depleted using a previously validated siRNA sequence ${ }^{27,28}$ (Figure 5 h), only MM-AN cells were significantly sensitized to TRAIL (Figure 5h). These results demonstrate that accurate predictions can be made on which proteins should be targeted to enhance apoptosis in cell lines that poorly respond to TRAIL.

\section{Discussion}

Cell death induction is a mainstay of anti-cancer chemotherapies $^{29}$ and is likely an important predictor if treatment modalities such as immune therapy can be effective to control disease relapse. Apoptosis is an irreversible cell fate decision and is regulated by the complex and nonlinear interplay of multiple proteins. Here, we integrated quantitative information on systems-level protein interplay with multivariate statistical 

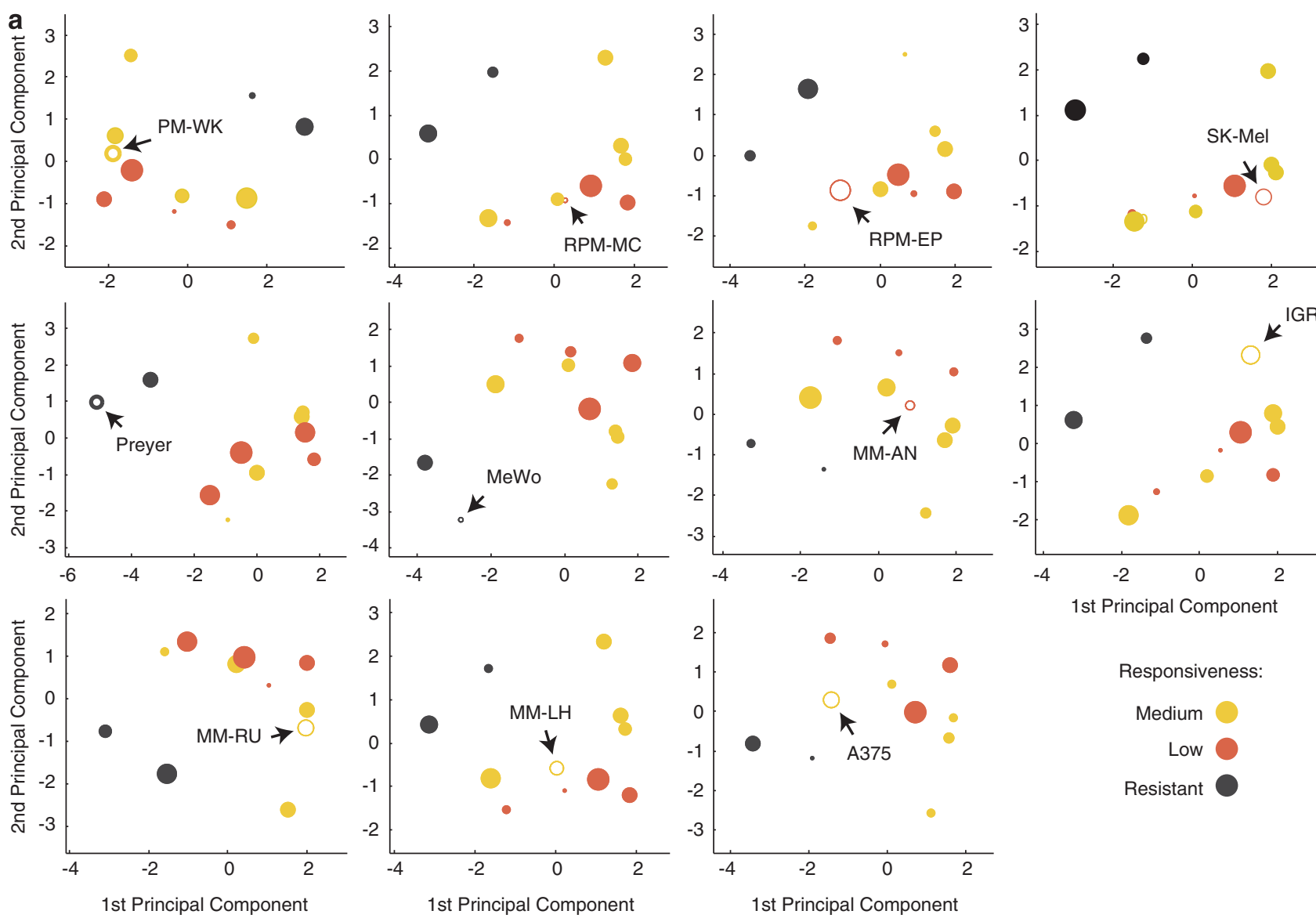

1st Principal Component

Responsiveness:

Medium

Low

Resistant

b Leave-one-out cross-validation:

\begin{tabular}{l|c|c} 
Drug & $\begin{array}{c}\text { Correctly } \\
\text { predicted \# }\end{array}$ & $\begin{array}{c}\text { Correctly } \\
\text { predicted in \% }\end{array}$ \\
\hline TRAIL & 10 & $91 \%$ \\
DTIC & 9 & $82 \%$
\end{tabular}

Figure 3 Systems analysis generates highly accurate case-specific predictions on TRAIL and DTIC responsiveness of melanoma cell lines. (a) Determination of predictive power by leave-one-out cross-validation (LOOCV). 2D projections of 3D PC spaces calculated from combinations of 10 cell lines are shown. LDA was applied to define spatial regions for resistant cell lines, low and medium responders for TRAIL treatment. Circle sizes decrease with distance, thereby representing the third PC dimension. Test cell lines (open circles, highlighted by arrows) were placed into the respective PC spaces according to their functional group values. If test cell lines positioned in spatial regions that were defined by prior LDA to correspond to their drug responsiveness, their responsiveness was considered to be predicted correctly. (b) Performance scores for correctly predicting drug responsiveness of test cell lines to TRAIL or DTIC by LOOCV-PCA and LDA in four-dimensional space

analysis and pattern recognition in order to generate predictions on the susceptibility of melanoma cells to TRAIL and DTIC (Figure 6). These predictions were highly accurate (91 and $82 \%$ correct predictions) and outperformed the predictive capacity of a systems knowledge-independent statistical analysis (55 and 36\% prediction accuracy). Our findings therefore indicate that to reliably predict cell death responsiveness the multi-factorial control of apoptosis signaling needs to be taken into account.

Our knowledge- and data-driven modeling approach was successfully applied for drug treatments that induce extrinsic and intrinsic apoptosis, and control measurements confirmed that apoptosis was the primary cell death modality (Supplementary Figure 3). Performance scores were very high for TRAIL-induced cell death, which may be attributable to our protein measurements having covered all critical TRAIL signal transducers. Furthermore, TRAIL-induced apoptosis can proceed independent of protein neosynthesis, explaining why a systems analysis of baseline protein expression is sufficient to predict TRAIL responsiveness. As TRAIL ligands are currently investigated in clinical trials and as improved second generation ligands are currently in preclinical development, ${ }^{30,31}$ our systems approach may provide possibilities to identify TRAIL-responsive melanoma by molecular profiling, and by extension may assist in patient stratification as part of future clinical trial designs. We also predicted DTIC-induced cell death, albeit with slightly lower accuracy. The induction of apoptosis by genotoxic stress is mediated by p53-dependent transcriptional induction of $\mathrm{BH}$-only proteins such as Noxa, Puma and Bik. ${ }^{32,33}$ Baseline expression amounts of these $\mathrm{BH}$-only proteins are typically low, and p53 is rarely mutated in melanoma $(<5 \%) .{ }^{34}$ This may explain 

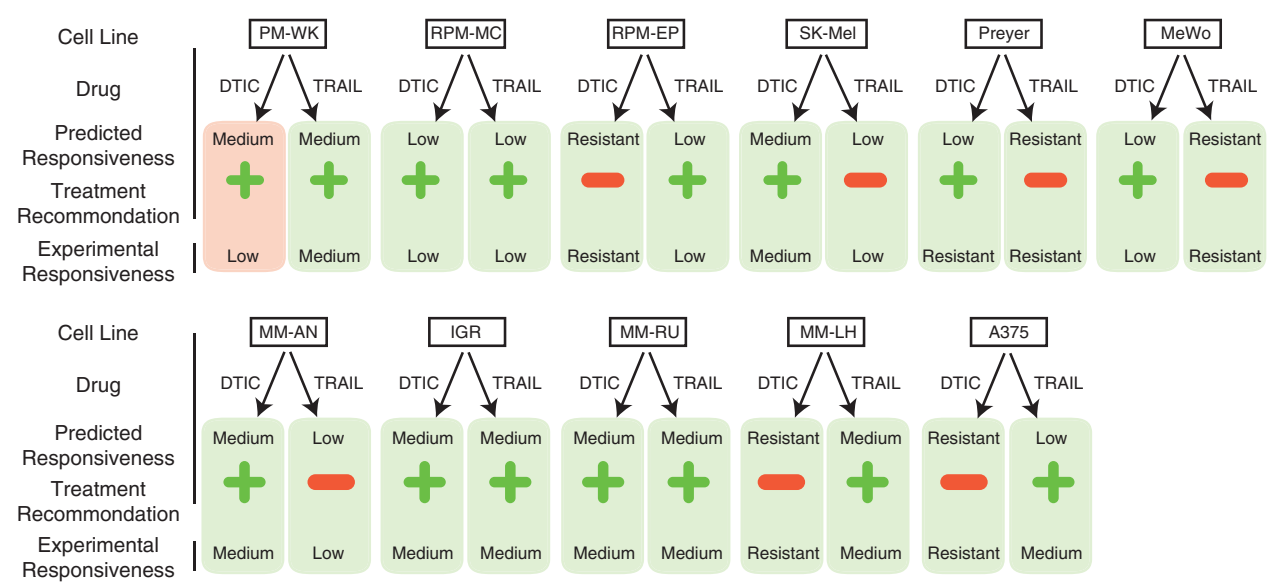

Figure 4 Case-specific predictions on TRAIL and DTIC responsiveness allow the in silico identification of optimal treatment options. The predictive capacity of the systems approach was exploited as a treatment decision tool. Treatment recommendations were made in silico according to highest predicted responsiveness to DTIC or TRAIL, respectively. Predictions were validated against experimental data. Predictions were labeled as correct (green) when the best treatment option could be identified, predictions were labeled as wrong (red) when the possibility existed that the better treatment option could be missed

why we achieved predictive capacity for DTIC treatments as well, even though we did not measure BH3-only protein amounts other than Bid. However, Bid has been reported to also contribute to genotoxic apoptosis, ${ }^{35}$ and in this context is cleaved by death receptor independent, presumably ripoptosome-activated caspase-8. ${ }^{36,37}$ A more comprehensive profiling of $\mathrm{BH} 3-$ only proteins may nevertheless further enhance the performance, and including information on the p53 mutational status could be required when developing this approach for other cancer entities. Likewise, coverage of alternative cell death mechanisms, such as necroptosis signaling, may be required for scenarios where apoptosisindependent cell death signaling has a substantial role. ${ }^{38,39}$

Various previous studies suggested that abundance measurements of proteins that regulate apoptosis in melanoma, including Bcl-2 family members and XIAP, ${ }^{40,41}$ may be sufficient to predict cell death susceptibility. We instead demonstrate that apoptosis susceptibility depends on the quantitative interplay of multiple proteins, and that targeted interventions to enhance responsiveness need to be identified on a case-by-case basis. Our study also highlights that because of the heterogeneity in apoptosis protein expression a single optimal treatment strategy cannot be suggested for a given tumor entity, exemplified here by malignant melanoma. As we investigated a large number of proteins $(n=17)$ in a large panel of cell lines $(n=11)$ by quantitative measurements, the biological heterogeneity in protein expression and cell death responsiveness in melanoma model systems may have been more representatively captured in our study than was the case in previous reports. Importantly, despite the heterogeneity in protein expression, the systems-level data analysis allowed insight into both drug responsiveness and resistance mechanisms of individual cell lines. Together, these findings may have important implications for biomarker identification strategies in the field of melanoma and other highly heterogeneous cancers. As the classical repertoire of preclinical and clinical biomarker screening so far yielded surprisingly few biomarkers that successfully translated into clinical practice, ${ }^{3}$ integrating systems-level approaches may contribute to improving this situation.
It can be speculated that our approach could be used to analyze primary tumors or metastases for their capacity to execute apoptosis, to predict therapy responsiveness and to generate personalized treatment recommendations. However, a validation for clinical application at the present time is challenging due to the need for large scale, quantitative protein data. Commonly used tissue immunohistochemistry in clinical diagnostics, based on immunoperoxidase staining, is of limited dynamic range, and stained tissue slides are typically not calibrated for quantitative readouts. Sufficient fresh frozen tissue as required for quantitative immunoblotting is rarely collected or available, and reverse phase protein arrays, which can be applied for large-scale multi-sample protein quantification in clinical specimens, ${ }^{42}$ require the prior identification or development of high-quality antibodies for each protein of interest. A successful clinical implementation of pathway-based systems models will furthermore necessitate their integration with mathematical models that cover higher level aspects known to influence therapy responsiveness. These include, for example, drug pharmacokinetics and pharmacodynamics, tumor vascularization and information on the tumor microenvironment and immune infiltration. Higher-scale modeling strategies that can make use of such information have been described in recent years ${ }^{10,43}$ and could be linked to models that have been validated in cellulo. Our study may therefore make an important contribution toward developing systems-based predictive tools that can be applied in a clinical context in the future.

\section{Materials and Methods}

Materials. DTIC was purchased from Sigma-Aldrich Ireland Ltd (Dublin, Ireland), ABT-737 was from Biorbyt (Riverside, UK), Mitotracker Red was from Invitrogen (Paisley, UK). Human recombinant TRAIL was a generous gift from Carlos Ricardo Rodrigues dos Reis, University of Groningen.

Cell culture and drug treatments. The human melanoma cell lines PM-WK, RPM-MC, RPM-EP, MM-RU, MM-AN and MM-LH were generously provided by Randy $\mathrm{H}$ Byers (Department of Dermatology, Boston University School of Medicine) and cultured as previously described. ${ }^{44}$ The following human melanoma cells were obtained either from ATCC (Manassas, VA, USA) or DSMZ (Deutsche Sammlung von Mikroorganismen und Zellkulturen GmbH, 
a

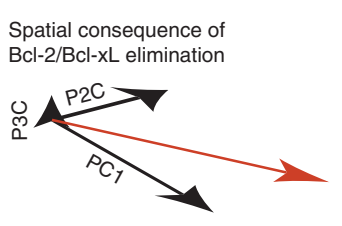

Spatial consequence of b

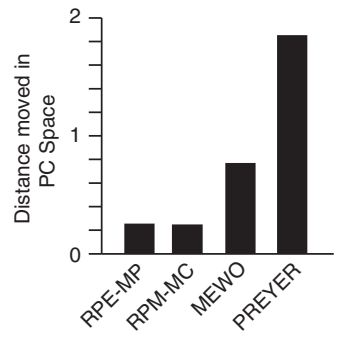

c

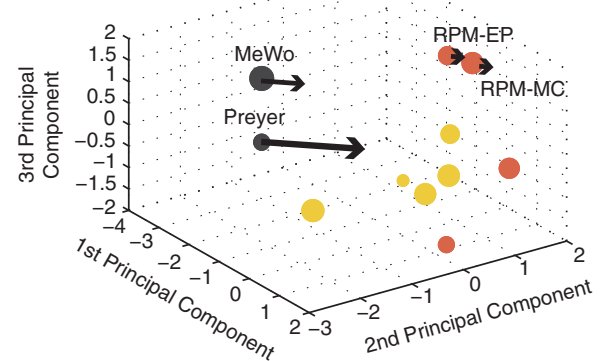

d

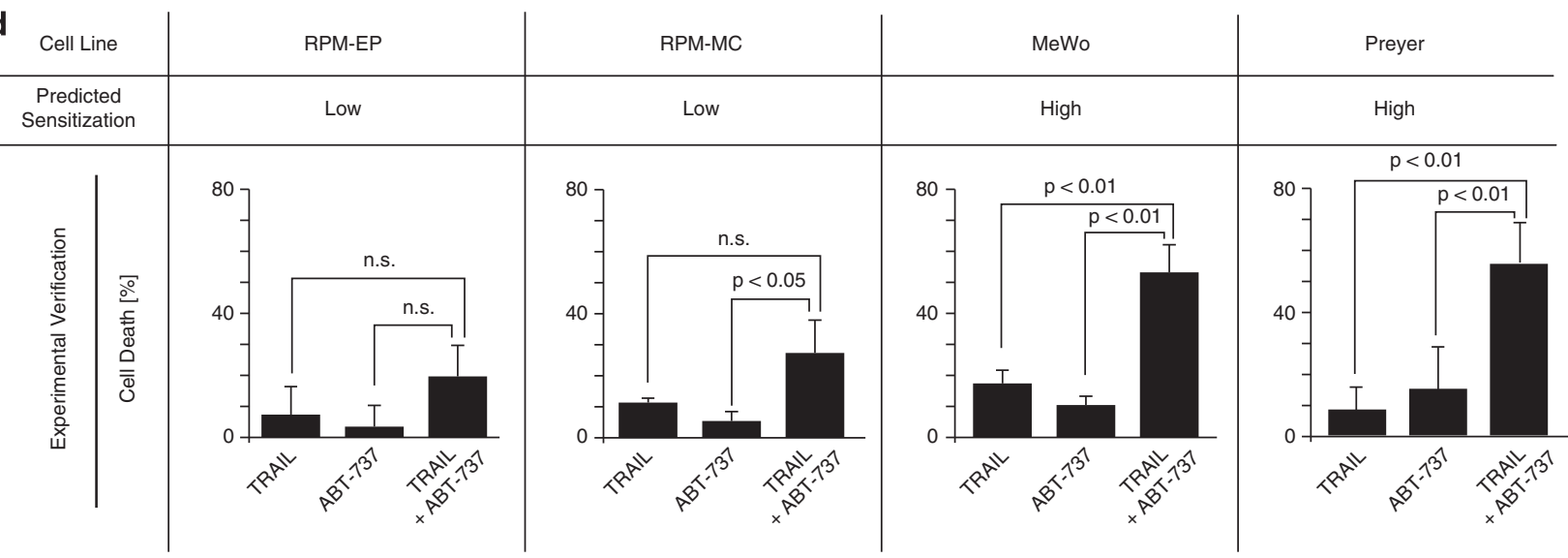

e Spatial consequence of XIAP elimination

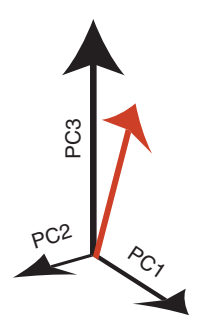

g

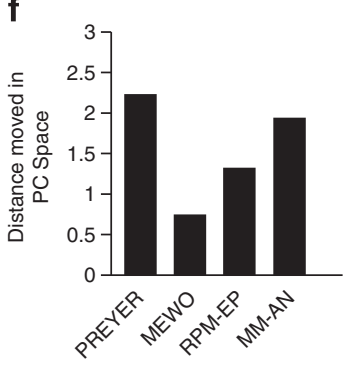

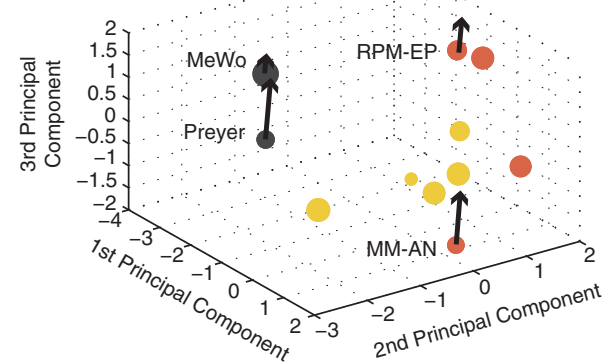

h

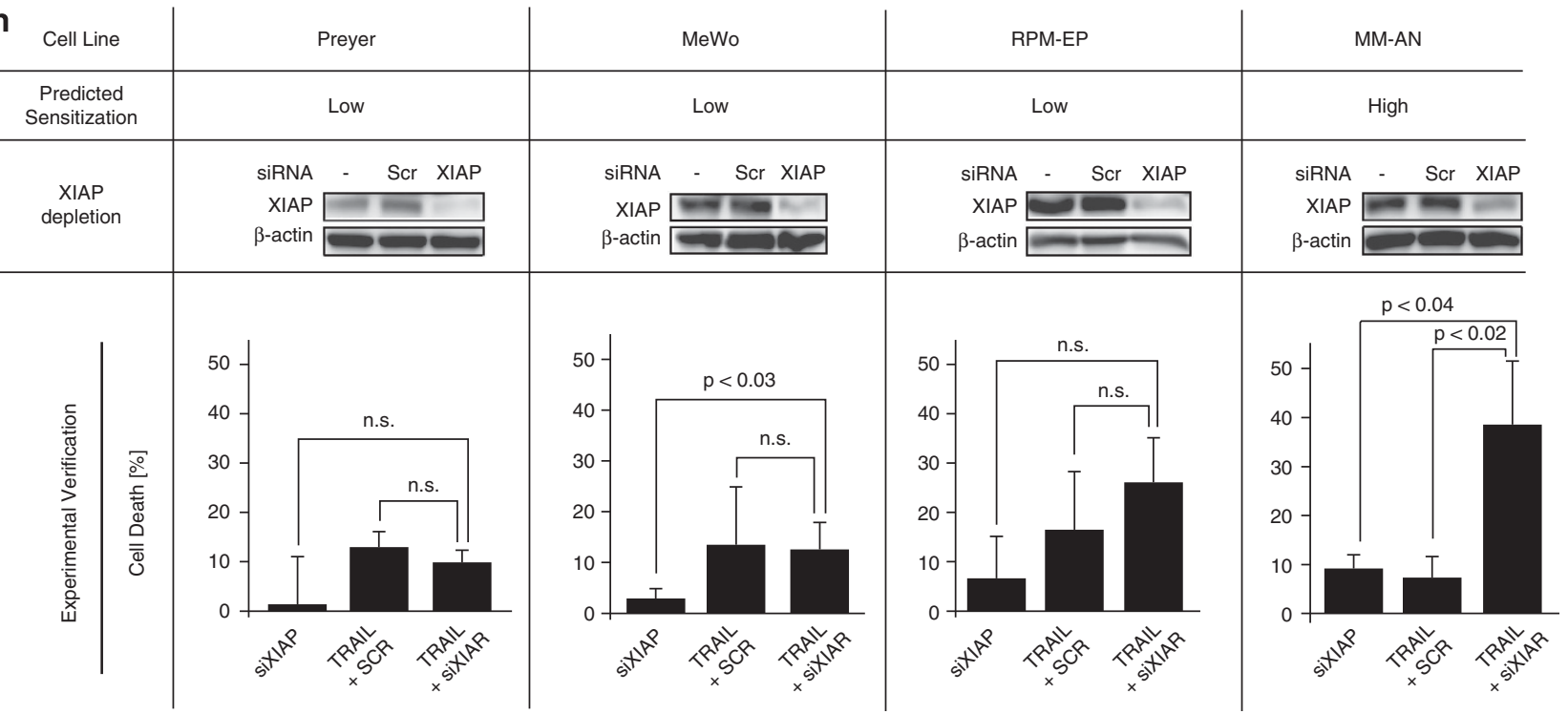


Braunschweig, Germany) and cultured as described: A375 (ATCC CRL-1619), MeWo (ATCC HTB-65), SK-Mel-30 (SK-Mel; ACC 151) and IGR-37 (IGR; ACC 237). Preyer melanoma cells (generated from a subcutaneous melanoma metastasis) were kindly provided by A Schwaaf and EB Bröcker (University of Würzburg, Germany). All melanoma cell lines were cultured in DMEM (Lonza, Slough, UK) supplemented with $4 \mathrm{mM}$ L-glutamine, $4.5 \mathrm{~g} / \mathrm{l}$ glucose, $10 \%$ (w/v) heatinactivated fetal bovine serum (Sigma-Aldrich), $100 \mathrm{U} / \mathrm{ml}$ penicillin and $100 \mu \mathrm{g} / \mathrm{ml}$ streptomycin (Sigma-Aldrich) as published. ${ }^{45}$ Cells were grown at $5 \% \mathrm{CO}_{2}$ and $37^{\circ} \mathrm{C}$. For cell death analysis, cells were incubated for $24 \mathrm{~h}$ with TRAIL $(100 \mathrm{ng} /$ $\mathrm{ml})$ or for $48 \mathrm{~h}$ with DTIC $(1 \mathrm{mg} / \mathrm{ml})$.

Transfections. siRNA known to specifically silence the expression of XIAP and non-silencing control sequences were obtained by Sigma-Aldrich (XIAP: $5^{\prime}$-AAGUGGUAGUCCUGUUUCAGC-3',28 control: $5^{\prime}$-UUCUCCGAACGUGUCAC GU[dT]-3'). Cells were transfected with siRNA $(100 \mathrm{nM})$ in Optimem with Lipofectamine 2000 (Invitrogen) according to the manufacturer's instruction at least $24 \mathrm{~h}$ before drug treatment. Protein depletion was validated by immunoblotting. Transient transfections for the expression of YFP-Bax were conducted as described before ${ }^{46}$ and cells were analyzed by conventional wide-field fluorescence microscopy.

Immunoblotting and densitometry. Cells were harvested, washed in PBS and homogenized in lysis buffer $(62.5 \mathrm{mM}$ Tris- $\mathrm{HCl}, \mathrm{pH} 6.8,10 \%(\mathrm{v} / \mathrm{v})$

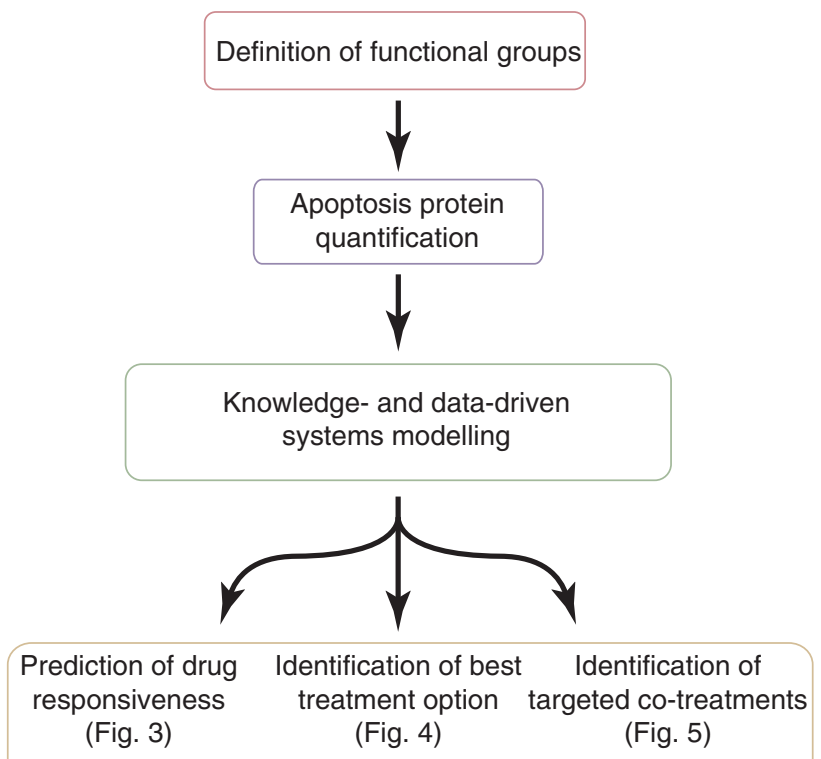

Figure 6 Workflow and associated functionalities for data- and knowledgedriven systems modeling to predict apoptosis sensitivity and treatment options. The systems analysis of apoptosis protein expression allowed (i) to predict responsiveness of melanoma cell lines to TRAIL and DTIC, (ii) to predict optimal treatment options for individual cell lines and (iii) to successfully identify targeted interventions that increased apoptosis responsiveness in treatment-resistant cells glycerin, $2 \%$ (w/v) SDS, $1 \mathrm{mM}$ phenylmethylsulfonyl fluoride, $1 \mu \mathrm{g} / \mathrm{ml}$ leupeptin and $5 \mathrm{~g} / \mathrm{ml}$ aprotinin). Lysates were heated to $95^{\circ} \mathrm{C}$ for $15 \mathrm{~min}$ and cleared of debris by centrifugation. Protein content was assayed using the Micro BCA protein assay kit (Thermo Scientific, Dublin, Ireland). Samples $(20 \mu \mathrm{g})$ were resolved on $10-15 \%$ SDS-polyacrylamide gels (150 V for approximately $1 \mathrm{~h}$ ). Proteins were transferred to nitrocellulose membranes (Whatman, Kent, UK) in transfer buffer (25 mM Tris, $192 \mathrm{mM}$ glycine, 20\% methanol (v/v) and 0.01 (w/v) SDS) at $18 \mathrm{~V}$ for $1.5 \mathrm{~h}$. Membranes were blocked in $5 \%$ nonfat dry milk in distilled water at room temperature for $1 \mathrm{~h}$ and incubated with primary antibodies overnight at $4{ }^{\circ} \mathrm{C}$. Membranes were then washed with TBST three times for $5 \mathrm{~min}$ and incubated with anti-mouse, anti-rabbit or anti-goat peroxidase-conjugated secondary antibodies (Millipore, Molsheim, France). The following primary antibodies were used: a rabbit polyclonal APAF-1 antibody (No. 559683, BD Transduction Laboratories, San Jose, CA, USA), a rabbit polyclonal Bak antibody (sc-832, Santa Cruz Biotechnology, Santa Cruz, CA, USA), a rabbit polyclonal Bax antibody (No. Q07815, Millipore), a mouse monoclonal Bcl-2 antibody (sc-509, Santa Cruz Biotechnology), a mouse monoclonal $\mathrm{Bcl}-\mathrm{X}_{\mathrm{L}}$ antibody (sc-8392, Santa Cruz Biotechnology), a goat polyclonal Bid antibody (AF860, R\&D Systems, Minneapolis, MN, USA), a rabbit polyclonal caspase-3 antibody (\#9662, Cell Signaling Technology, Danvers, MA, USA), a mouse monoclonal caspase-8 antibody (No 804-242, Alexis, San Diego, CA, USA), a rabbit polyclonal caspase-9 antibody (No. 218794, Calbiochem/Merck Bioscience, Nottingham, UK), a mouse monoclonal cyt-c antibody (No. 556433, BD Transduction Laboratories), a mouse monoclonal DR4 antibody (ab47138, AbCam, Cambridge, UK), a rabbit polyclonal DR5 antibody (No 804-298, Alexis), a mouse monoclonal FADD antibody (No. 610399, BD Transduction Laboratories), a mouse monoclonal FLIP antibody (No 804-428, Alexis), a mouse monoclonal Mcl-1 antibody (No. 559027, BD Transduction Laboratories), a rabbit polyclonal SMAC/Diablo antibody (AF789, R\&D Systems), a mouse monoclonal XIAP antibody (No 610763, BD Transduction Laboratories), a mouse monoclonal $\beta$-actin antibody (A5441, Sigma-Aldrich). Antimouse $\lg G$, anti-goat $\lg G$, anti-rabbit $\lg G$ peroxidise-conjugated secondary antibodies (AP124P, AP106P, AP132P, Millipore) were used at a dilution of 1:5000 for $1 \mathrm{~h}$. Blots were washed and developed using the Immobilon western chemiluminescence HRP substrate (Millipore). Chemiluminescence was detected at a depth of 12-bit in the linear detection range of a Fuji LAS 4000 CCD system (Fujifilm UK Ltd, Bedfordshire, UK). Special care was taken not to overexpose in order to guarantee accurate quantifications. For all proteins, at least three independent membranes were analyzed. Densitometry was performed on 12-bit raw images using ImageJ 1.4.10 software (National Institute of Health, Bethesda, MD, USA; http://rsb.info.nih.gov/ij). ${ }^{47}$ For each protein, the integrated density of the signal was measured, corrected for background signals and adjusted to loading controls. Intensities were then compared with signals obtained from HeLa cell extracts run on the same gels. Absolute protein levels in HeLa cells (nM) have been determined by us and others before by comparison to titrated purified proteins. $^{47,48}$ For visual presentation, 12-bit images were contrast adjusted and converted to 8-bit.

Flow cytometry. Flow cytometry was performed on a BD LSRIl flow cytometer (BD Bioscience, Oxford, UK) equipped with a high-throughput system to acquire samples directly from 96 -well plates. DR-4 and DR-5 surface expression were assessed by indirect immunofluorescence. Briefly, cells were harvested, washed, fixed in $4 \%$ paraformaldehyde in PBS for 15 min and incubated with a mouse monoclonal DR4 (AbCam) or DR5 antibody (Alexis; $5 \mu \mathrm{g} / 10^{6}$ cells) for $30 \mathrm{~min}$ followed by secondary anti-mouse FITC-conjugated antibody (AbCam; 1:50 dilution) for $30 \mathrm{~min}$ in the dark. Controls were stained with secondary antibody

Figure 5 Prediction and experimental validation of targeted perturbations that sensitize poorly responding cell lines to TRAIL. (a-d) Targeted perturbation with Bcl-2/Bcl$\mathrm{XL}$ antagonist ABT-737. (a) Inverse vectorial addition of the coefficients of the $\mathrm{Bcl}-2+\mathrm{Bcl}-\mathrm{xL}+\mathrm{Mcl}-1$ functional group in the first three PCs. The resultant vector is shown in red and indicates the direction of cell line displacement in the PC space upon antagonizing this functional group by ABT-737. (b) Bar graphs show the distance by which individual cell lines are displaced in the 3D PC space when neutralizing Bcl-2 and Bcl-xL by ABT-737. (c) Combination of movement direction and distance shown in the 3D PC space for four poorly TRAIL-responsive cell lines. (d) Experimental validation of sensitization predictions. Cells were treated with $100 \mathrm{ng} / \mathrm{ml}$ TRAIL and/or $5 \mu$ M ABT-737 as indicated. Data show cell death above untreated controls (means + S.D. from $n=3$ independent experiments). Student's t-test was used for statistical analysis. (e-h) Targeted perturbation by siRNA-mediated XIAP depletion. (e) The red vector indicates the direction of cell line displacement by XIAP depletion. (f) Bar graphs indicate the distance of movement of individual cell lines in the 3D PC space upon eliminating XIAP. (g) Combination of movement direction and distance shown in the 3D PC space for four poorly TRAIL-responsive cell lines. (h) Experimental validation of sensitization predictions. Cells were treated with $100 \mathrm{ng} / \mathrm{ml}$ TRAIL $24 \mathrm{~h}$ after transfection with $100 \mathrm{nM}$ XIAP siRNA or scrambled control siRNA. Data show cell death above controls transfected with scrambled siRNA (means + S.D. from $n=3$ independent experiments). Student's t-test was used for statistical analysis. Immunoblot insets show the efficacy of XIAP depletion by siRNA 
only. After each incubation, cells were washed with $3 \%$ BSA in PBS to remove excess antibody. FITC was excited at $488 \mathrm{~nm}$ and fluorescence emission was collected through a 552/50-nm band-pass filter and a 505-nm long pass filter. The relative expression of death receptors was determined by comparison of specific staining intensities. For cell death measurements, cells were detached and incubated on an orbital shaker (300 r.p.m.) with propidium iodide $(2 \mu \mathrm{g} / \mathrm{ml}$; Sigma-Aldrich) at room temperature for $15 \mathrm{~min}$ in the dark. Propidium iodide was excited with a 561-nm laser line and fluorescence emission was collected through a 605/40-nm band-pass filter and a 570-nm long pass filter. Data were analyzed using Cyflogic software (CyFlo Ltd, Turku, Finland). Cell death measurements were conducted as end-point readings. Control experiments in selected high- and low-responding cell lines were performed at multiple time points with AnnexinVFITC (Biovision, Milpitas, CA, USA)/propidium iodide co-staining to ensure the absence of significant amounts of early apoptotic cells (Annexin V $+/ P I-$ ) at the time of measurement.

Data processing and analysis for knowledge- and data-driven modeling. All data processing and analysis were performed using a programming code developed for MATLAB 2007b (The Mathworks, Cambridge, UK), equipped with the statistics toolbox. Protein data in functional groups were rescaled before statistical analysis by dividing through the respective standard deviation, followed by mean centering. A PCA based on the correlation matrix of these data was performed. ${ }^{19,20}$ The Kaiser criterion and an adapted scree plot were used to identify how many PCs needed to be retained for subsequent analyses. ${ }^{21,22}$ For visualizations, scatter plots were generated from the first three PCs. Cell lines were assigned to classes of cell death responsiveness using k-means clustering. LDA ${ }^{24}$ was performed in the first four PCs to determine the quality of response class separation in the PC space. To determine predictive capacity, LOOCV was applied iteratively on training sets of 10 cell lines, with the remaining cell line being the test set. The test cell line was placed into the PC space according to its specific functional group values and associated PC coefficients calculated from the training cell lines. LDA was applied to determine whether the test cell line positioned in the spatial region that corresponded to its drug responsiveness. To predict the consequences of targeted protein perturbations, the target protein concentrations were set to zero, and the displacement of individual cell lines was calculated by multiplying the changes in the respective functional groups with the coefficients in the first three PCs. Movement toward spatial regions of higher responsiveness were considered to be predictive of sensitization.

Statistical testing. Student's t-tests were used to identify statistically significant differences between treatment groups. $P$-values above 0.05 were considered not to be significant. $P$-value ranges below 0.05 were indicated in the respective figure panels. Analyses were performed using SPSS 15 (Lead Technologies Inc., Charlotte, NC, USA).

\section{Conflict of Interest}

The authors declare no conflict of interest.

Acknowledgements. We thank Carlos Ricardo Rodrigues dos Reis, University of Groningen, for providing human recombinant TRAIL and Frank A Lincoln for technical assistance. This research was supported by grants from the Health Research Board Ireland (RP/2008/7), the National Biophotonics and Imaging Platform (HEA PRTLI Cycle 4) and the EU Framework Programme 7 (APO-DECIDE). The work in the laboratory of ML is supported by grants from the DFG (Le 953/6-1 and 8-1), the Wilhelm-Sander-Stiftung (2008.072.1) and the Mildred-Scheel-Stiftung (Projekt 109891).

1. Lui P, Cashin R, Machado M, Hemels M, Corey-Lisle PK, Einarson TR. Treatments for metastatic melanoma: synthesis of evidence from randomized trials. Cancer Treat Rev 2007; 33: 665-680

2. Paul SM, Mytelka DS, Dunwiddie CT, Persinger CC, Munos BH, Lindborg SR et al. How to improve R\&D productivity: the pharmaceutical industry's grand challenge. Nat Rev Drug Discov 2010; 9: 203-214.

3. Poste G. Bring on the biomarkers. Nature 2011; 469: 156-157.
4. Chapman PB, Hauschild A, Robert C, Haanen JB, Ascierto P, Larkin J et al. Improved survival with vemurafenib in melanoma with BRAF V600E mutation. N Engl J Med 2011; 364: 2507-2516.

5. Wolchok JD, Kluger H, Callahan MK, Postow MA, Rizvi NA, Lesokhin AM et al. Nivolumab plus Ipilimumab in Advanced Melanoma. N Engl J Med 2013; 369: 122-133.

6. Soengas MS, Lowe SW. Apoptosis and melanoma chemoresistance. Oncogene 2003; 22: 3138-3151.

7. Hellwig CT, Passante E, Rehm M. The molecular machinery regulating apoptosis signal transduction and its implication in human physiology and pathophysiologies. Curr Mol Med 2011; 11: 31-47.

8. Taylor RC, Cullen SP, Martin SJ. Apoptosis: controlled demolition at the cellular level. Nat Rev Mol Cell Biol 2008; 9: 231-241.

9. Auffray C, Chen Z, Hood L. Systems medicine: the future of medical genomics and healthcare. Genome Med 2009; 1: 2

10. Gorelik B, Ziv I, Shohat R, Wick M, Hankins WD, Sidransky D et al. Efficacy of weekly docetaxel and bevacizumab in mesenchymal chondrosarcoma: a new theranostic method combining xenografted biopsies with a mathematical model. Cancer Res 2008; 68: 9033-9040.

11. Hector S, Rehm M, Schmid J, Kehoe J, McCawley N, Dicker P et al. Clinical application of a systems model of apoptosis execution for the prediction of colorectal cancer therapy responses and personalisation of therapy. Gut 2012; 61: 725-733.

12. Waterhouse NJ, Goldstein JC, von Ahsen O, Schuler M, Newmeyer DD, Green DR. Cytochrome $\mathrm{c}$ maintains mitochondrial transmembrane potential and ATP generation after outer mitochondrial membrane permeabilization during the apoptotic process. $J$ Cell Biol 2001; 153: 319-328.

13. Quinn PJ, Dawson RM. Interactions of cytochrome c and [14C]-carboxymethylated cytochrome $\mathrm{c}$ with monolayers of phosphatidylcholine, phosphatidic acid and cardiolipin. Biochem J 1969; 115: 65-75.

14. Ott M, Zhivotovsky B, Orrenius S. Role of cardiolipin in cytochrome $\mathrm{c}$ release from mitochondria. Cell Death Differ 2007; 14: 1243-1247.

15. Chandra D, Bratton SB, Person MD, Tian Y, Martin AG, Ayres M et al. Intracellular nucleotides act as critical prosurvival factors by binding to cytochrome $\mathrm{C}$ and inhibiting apoptosome. Cell 2006; 125: 1333-1346.

16. Mei $Y$, Yong J, Liu H, Shi Y, Meinkoth J, Dreyfuss $\mathrm{G}$ et al. tRNA binds to cytochrome $\mathrm{c}$ and inhibits caspase activation. Mol Cell 2010; 37: 668-678.

17. Vaughn AE, Deshmukh M. Glucose metabolism inhibits apoptosis in neurons and cancer cells by redox inactivation of cytochrome c. Nat Cell Biol 2008; 10: 1477-1483.

18. Janes KA, Yaffe MB. Data-driven modelling of signal-transduction networks. Nat Rev Mol Cell Biol 2006; 7: 820-828.

19. Hotelling $\mathrm{H}$. Analysis of a complex of statistical variables into principal components. J Educ Psych 1933; 24: 498-520.

20. Pearson K. On lines and planes of closest fit to systems of points in space. Philos Mag 1901; 2: 559-572.

21. Kaiser HF. The Application of electronic computers to factor analysis. Educ Psychol Meas 1960; 20: 141-151.

22. Cattell RB. The scree test for the number of factors. Multivariate Behav Res 1966; 1: 245-276.

23. Reid JM, Kuffel MJ, Miller JK, Rios R, Ames MM. Metabolic activation of dacarbazine by human cytochromes P450: the role of CYP1A1, CYP1A2, and CYP2E1. Clin Cancer Res 1999; 5: 2192-2197.

24. Fisher RA. The use of multiple measurements in taxonomic problems. Ann Hum Genet 1936; $7: 179-188$

25. Oltersdorf T, Elmore SW, Shoemaker AR, Armstrong RC, Augeri DJ, Belli BA et al. An inhibitor of Bcl-2 family proteins induces regression of solid tumours. Nature 2005; 435 : $677-681$.

26. Cragg MS, Jansen ES, Cook M, Harris C, Strasser A, Scott CL. Treatment of B-RAF mutant human tumor cells with a MEK inhibitor requires $\mathrm{Bim}$ and is enhanced by a $\mathrm{BH} 3$ mimetic. J Clin Invest 2008; 118: 3651-3659.

27. Laussmann MA, Passante E, Dussmann H, Rauen JA, Wurstle ML, Delgado ME et al. Proteasome inhibition can induce an autophagy-dependent apical activation of caspase-8. Cell Death Differ 2011; 18: 1584-1597.

28. Wilkinson JC, Cepero E, Boise LH, Duckett CS. Upstream regulatory role for XIAP in receptor-mediated apoptosis. Mol Cell Biol 2004; 24: 7003-7014.

29. Hanahan D, Weinberg RA. Hallmarks of cancer: the next generation. Cell 2011; 144: 646-674.

30. Hellwig CT, Rehm M. TRAIL signaling and synergy mechanisms used in TRAlL-based combination therapies. Mol Cancer Ther 2012; 11: 3-13.

31. Wilson NS, Yang B, Yang A, Loeser S, Marsters S, Lawrence D et al. An Fcgamma receptor-dependent mechanism drives antibody-mediated target-receptor signaling in cancer cells. Cancer Cell 2011; 19: 101-113.

32. Weber A, Kirejczyk Z, Potthoff S, Ploner C, Hacker G. Endogenous Noxa determines the strong proapoptotic synergism of the BH3-mimetic ABT-737 with chemotherapeutic agents in human melanoma cells. Trans Oncol 2009; 2: 73-83.

33. Cory S, Adams JM. The Bcl2 family: regulators of the cellular life-or-death switch. Nat Rev Cancer 2002; 2: 647-656.

34. Lubbe J, Reichel M, Burg G, Kleihues P. Absence of p53 gene mutations in cutaneous melanoma. J Invest Dermatol 1994; 102: 819-821. 
35. Kohler B, Anguissola S, Concannon CG, Rehm M, Kogel D, Prehn JH. Bid participates in genotoxic drug-induced apoptosis of HeLa cells and is essential for death receptor ligands' apoptotic and synergistic effects. PLoS One 2008; 3: e2844.

36. Tenev T, Bianchi K, Darding M, Broemer M, Langlais C, Wallberg F et al. The ripoptosome, a signaling platform that assembles in response to genotoxic stress and loss of IAPs. $\mathrm{Mol}$ Cell 2011; 43: 432-448.

37. Feoktistova M, Geserick P, Kellert B, Dimitrova DP, Langlais $C$, Hupe M et al. clAPs block ripoptosome formation, a RIP1/caspase-8 containing intracellular cell death complex differentially regulated by cFLIP isoforms. Mol Cell 2011; 43: 449-463.

38. Kreuzaler P, Watson CJ. Killing a cancer: what are the alternatives? Nat Rev Cancer 2012 12: $411-424$.

39. Vanlangenakker $N$, Vanden Berghe $T$, Vandenabeele $P$. Many stimuli pull the necrotic trigger, an overview. Cell Death Differ 2012; 19: 75-86.

40. Tang L, Tron VA, Reed JC, Mah KJ, Krajewska M, Li G et al. Expression of apoptosis regulators in cutaneous malignant melanoma. Clin Cancer Res 1998; 4 : 1865-1871.

41. Hiscutt EL, Hill DS, Martin S, Kerr R, Harbottle A, Birch-Machin M et al. Targeting X-linked inhibitor of apoptosis protein to increase the efficacy of endoplasmic reticulum stressinduced apoptosis for melanoma therapy. J Invest Dermatol 2010; 130: 2250-2258.

42. Mueller C, Liotta LA, Espina V. Reverse phase protein microarrays advance to use in clinical trials. Mol Oncol 2010; 4: 461-481.
43. Eissing T, Kuepfer L, Becker C, Block M, Coboeken K, Gaub T et al. A computational systems biology software platform for multiscale modeling and simulation: integrating whole-body physiology, disease biology, and molecular reaction networks. Front Physiol 2011; $2: 4$.

44. Byers HR, Etoh T, Doherty JR, Sober AJ, Mihm MC Jr. Cell migration and actin organization in cultured human primary, recurrent cutaneous and metastatic melanoma. Time-lapse and image analysis. Am J Pathol 1991; 139: 423-435.

45. Geserick P, Drewniok C, Hupe M, Haas TL, Diessenbacher P, Sprick MR et al Suppression of CFLIP is sufficient to sensitize human melanoma cells to TRAIL- and CD95L-mediated apoptosis. Oncogene 2008; 27: 3211-3220.

46. Dussmann H, Rehm M, Concannon CG, Anguissola S, Wurstle M, Kacmar S et al. Single-cell quantification of Bax activation and mathematical modelling suggest pore formation on minimal mitochondrial Bax accumulation. Cell Death Differ 2010; 17: 278-290.

47. Rehm M, Huber HJ, Dussmann H, Prehn JH. Systems analysis of effector caspase activation and its control by $\mathrm{X}$-linked inhibitor of apoptosis protein. EMBO J 2006; 25: 4338-4349.

48. Lindner AU, Concannon CG, Boukes GJ, Cannon MD, Llambi F, Ryan D et al. Systems analysis of $\mathrm{BCL} 2$ protein family interactions establishes a model to predict responses to chemotherapy. Cancer Res 2013; 73: 519-528.

Supplementary Information accompanies this paper on Cell Death and Differentiation website (http://www.nature.com/cdd) 